\title{
The use of platelet-rich plasma to augment conservative and surgical treatment of hip and pelvic disorders
}

\author{
Matthew J. Kraeutler ${ }^{1}$ \\ Tigran Garabekyan ${ }^{2}$ \\ Omer Mei-Dan ${ }^{3}$ \\ 1 University of Colorado School of Medicine, \\ Department of Orthopedics, Aurora, USA \\ 2 Southern California Hip Institute, North Hollywood, \\ CA, USA \\ 3 Hip Preservation/ Sports Medicine/Orthopedics \\ University of Colorado
}

Corresponding author:

Omer Mei-Dan

Hip Preservation/Sports Medicine/Orthopedics

University of Colorado

12631 East 17th Avenue

Mail Stop B202, Room L15-4505

Aurora, Colorado 80045 (USA)

E-mail: omer.meidan@ucdenver.edu

\section{Summary}

Background: In recent years, platelet-rich plasma (PRP) has gained popularity within the orthopaedic community as a treatment modality to enhance tissue healing.

Purpose: This review aims to concisely present the current indications for PRP injections in the treatment of hip and pelvic pathologies and to describe some novel applications for PRP which have not yet been reported in the literature.

Methods: We reviewed the literature on the non-operative and operative indications for PRP in the treatment of hip and pelvic pathologies.

Conclusions: With regard to hip and pelvic pathologies, PRP injections are used most commonly as a non-operative intervention, and have been described in the literature to treat osteoarthritis of the hip joint as well as tendinopathy of the hamstrings, adductor longus, and gluteus medius. In contrast, most of the surgical applications of PRP for the hip are novel, with few reported studies in the literature. Because of the increasing awareness of PRP's beneficial effects on musculoskeletal healing and thus the growing number of indications for its use, this review also describes some novel applications for PRP, including osteitis pubis, post-microfracture of the hip, tears of the rectus femoris, and avulsion of the sartorius muscle.

Level of evidence: $\mathrm{V}$.
KEY WORDS: hamstring tendinopathy, hip osteoarthritis, osteitis pubis, microfracture, platelet-rich plasma.

\section{Introduction}

Platelet-rich plasma (PRP) has gained popularity within the last decade among the orthopaedic community as a treatment modality to enhance tissue healing. The term platelet-rich plasma may be applied to any fraction of autologous blood that contains a higher concentration of platelets than baseline ${ }^{1}$. Thus, this term is non-specific and factors such as the concentration of platelets and leukocytes as well as centrifugation methods have differed between studies. DeLong et al. ${ }^{2}$ developed the PAW classification system to aid in comparing different protocols of PRP preparation. This classification system is based on the absolute number of platelets $(P)$, the method of platelet activation $(A)$, and the presence/absence of white cells (W).

Recently, PRP has been utilized for numerous musculoskeletal indications such as rotator cuff repair ${ }^{3,4}$, patellar tendinopathy ${ }^{5}$, knee osteoarthritis ${ }^{6}$, lateral epicondylitis $^{7}$, osteochondral lesions of the talus ${ }^{8}$, and many other orthopaedic conditions. PRP induces musculoskeletal healing through a number of effects. As a treatment modality for tendon healing, PRP enhances the mobilization of circulation-derived cells ${ }^{9}$. This may include inflammatory cells that secrete cytokines and growth factors as well as fibroblast-like cells that synthesize matrix. Compared to serum, PRP has been shown to significantly increase the deposition of a collagen-rich extracellular matrix ${ }^{10}$, with higher collagen I content compared to placebo ${ }^{11}$. Interestingly, PRPtreated tendon tears have actually been shown to contain fewer blood vessels compared to placebo ${ }^{11}$, possibly indicating a more physiological healing process. Once PRP enhances the early phase of regeneration, mechanical stimulation is required to promote organized collagen synthesis and remodeling during new tendon development ${ }^{12}$.

With muscle strains or contusions, the hematoma that originates contains approximately $94 \%$ red blood cells, $4 \%$ platelets, and $<1 \%$ leukocytes ${ }^{13}$. Compared to whole blood, PRP contains higher concentrations of certain growth factors, in particular platelet-derived growth factor (PDGF) and transforming growth factor- $\beta$ $(\text { TGF }-\beta)^{14}$. Thus, PRP is theorized to replace the hematoma with a high concentration of platelets and growth factors to promote healing. Furthermore, PRP has been shown to promote angiogenesis through activation of PRP-releasate (PRP-r) ${ }^{15}$. In comparison to 
tendon and muscle healing, little is known on the mechanisms of PRP in promoting healing of articular cartilage, though this likely involves multiple biological processes including apoptosis, extracellular matrix synthesis, angiogenesis, and inflammation ${ }^{16}$. Because of the increasing awareness of PRP's beneficial effects on musculoskeletal healing and thus the growing number of indications for its use, we present a review of the current indications for platelet-rich plasma injections to augment the conservative and surgical treatment of hip and pelvic pathologies and describe some novel applications for PRP which have not yet been described in the literature. Although several studies have described the use of PRP for some of these pathologies, other indications for PRP discussed in this review have not been published previously. As such, these indications are not yet evidence-based. This article submits to the ethical standards of the journal ${ }^{17}$.

\section{Decision-making}

After performing an appropriate patient history and physical examination, advanced imaging is typically obtained to better characterize the suspected pathology. With muscle or tendon tears, magnetic resonance imaging (MRI) or ultrasound (US) should be used to determine the exact location and extent of the injury. Depending on the pathology, PRP may be used as a conservative treatment measure or as adjunctive treatment during surgery. When used as a conservative treatment option, PRP may be applicable for tendinopathic changes or partial tendon tears in which the tendon ends are not retracted ${ }^{1}$

The cost of platelet-rich plasma treatment is certainly a factor in the decision-making process. Insurance companies still do not recognize PRP as standard of care, and thus PRP must be paid by patients out of pocket. It has been estimated recently that the cost of PRP is $\$ 500$ to $\$ 1,500$ per application ${ }^{18}$. It is important to have an open discussion regarding the cost of PRP injections, given that it may be prohibitively expensive for some patients.

\section{Preparation and application techniques}

A sample of whole blood is collected in a sodium citrate tube in order to delay clotting of the blood sample. Once the whole blood sample is collected, centrifugation allows separation of the sample into its component cells and serum. Either one or two centrifugation steps may be used depending on the final product desired. A "soft" spin separates the whole blood sample into three layers: an upper layer consisting mainly of plasma and platelets, a very thin middle layer known as the "buffy coat" that is highly concentrated in WBCs, and a bottom layer consisting mainly of red blood cells (RBCs) ${ }^{19}$. A second, "hard" spin may be used to further concentrate the platelets ${ }^{19,20}$. Following centrifugation, approximately $10 \%$ of the initial whole blood volume remains as PRP concentrate ${ }^{1}$. The platelets in the PRP concen- trate are activated with calcium chloride and/or autologous or bovine thrombin. These additions are used to initiate the clotting cascade, the release of growth factors from the platelets, and the formation of a fibrin scaffold $^{20}$. Autologous thrombin has been shown to have a lower clot strength compared to bovine thrombin or calcium chloride, with bovine thrombin having the quickest clot initiation time ${ }^{21}$. In an equine model, calcium chloride activation of PRP has been shown to result in greater release of platelet-derived growth factor compared to autologous or bovine thrombin ${ }^{22}$. Calcium chloride also provides the advantage of not using bovine or other non-autologous materials.

Therapeutic doses of PRP require 2.5-5 times the baseline concentration of platelets ${ }^{23,24}$, though higher concentrations than this have an inhibitory effect on healing ${ }^{25}$. The white blood cell concentration may also be controlled, with leukocyte-rich PRP (L-PRP) and leukocyte-poor PRP (P-PRP) both being used in the literature. For production of L-PRP, the entire layer of the buffy coat and few RBCs are transferred to an empty sterile tube, while the upper layer and only the superficial buffy coat are transferred for production of $P$ $\mathrm{PRP}^{19}$. Plasma rich in growth factors (PRGF) is a term used to describe a leukocyte-poor PRP which is separated manually (direct visualization using a fine pipette) from the lower fraction of the plasma containing the highest concentration of platelets and growth factors, avoiding the thin WBC layer. PRGF techniques have been shown to produce lower concentrations of growth factors compared to standard PRP kits by 3-4 fold26 which, according to many studies, may serve as the optimal ratio for tissue healing.

When a tendon or muscle is injured, healing proceeds through three processes: inflammation/degeneration, regeneration, and fibrosis ${ }^{1}$. Although L-PRP has been shown to contain the highest levels of growth factors and cytokines ${ }^{27}$, it induces catabolic effects and a significantly greater acute inflammatory response and thus may actually prolong the healing process ${ }^{27-30}$. Thus, the inclusion of white cells defeats the purpose of PRP. On the other hand, P-PRP induces mainly anabolic changes, and while this is generally a beneficial outcome, it could also result in scar tissue formation due to these anabolic effects 27,30 . Still, no randomized or prospective clinical studies have been performed to compare outcomes between leukocyte-rich versus leukocyte-poor PRP.

\section{Non-operative applications}

Platelet-rich plasma injections are used most commonly as an adjunct to conservative treatment. In cases of chronic tendinopathy or osteoarthritis, PRP is typically indicated when first-line treatment (physical therapy, rest) fails. For professional athletes, in-season PRP may be used to reduce pain and improve function as an interim solution until the offseason when the athlete can undergo surgical intervention. This is particularly true for hip labral tears and chronic tendinopathies.

As detailed below, PRP injections have been used to 


\section{J. Kraeutler et al.}

treat tendinopathy of the hamstrings, adductor longus, and gluteus medius. These injections are typically performed under US guidance in the clinic. The number of injections used differs by study, though most reports describe the use of a single PRP injection for a variety of hip pathologies. Prior to injection, patients should fast for a minimum of three hours and should limit water intake to 8 ounces. In addition, patients should avoid the use of non-steroidal anti-inflammatory drugs (NSAIDs) for at least two days prior to and five days following injection, as these medications have been shown to impair platelet function ${ }^{31}$

\section{Hamstring tendinopathy}

Chronic tendinopathy or partial tears of the proximal hamstring tendons are common injuries among athletes. These injuries often occur while running, particularly when accelerating. Following severe hamstring injuries, many high level athletes may struggle with sitting on a bike or returning to running. Corticosteroid injections should be avoided as they may result in further tendon weakening and progression to high-grade tearing. PRP injections can be used to facilitate healing when there is partial thickness involvement or tendinosis without retraction. Complete tears, especially if chronic and retracted, are best treated with surgical repair.
A Hamid et al. ${ }^{32}$ conducted a randomized controlled trial to compare PRP therapy plus a rehabilitation program versus rehabilitation alone in patients with acute hamstring injuries (Table I). Patients in the PRP group were given a single intra-lesional injection of PRP, without addition of an activating agent, under US guidance at an average of 4.6 days following injury. Time to return to play was significantly lower in the PRP group (mean 26.7 days) versus the control group (mean 42.5 days). In addition, the PRP group had significantly lower pain severity scores at all time points up to 7 weeks following intervention.

In another randomized controlled trial, Hamilton et al. ${ }^{33}$ compared PRP, platelet-poor plasma (PPP), and no injection in professional athletes with MRI-positive hamstring injuries. All patients underwent a standardized rehabilitation program. Time to return to sport was found to be significantly lower in the PRP group (mean 21 days) compared with the PPP group (mean 27 days). No significant difference in re-injury rate was noted between the three groups at 2 months or 6 months following intervention.

Fader et al. ${ }^{34}$ performed a retrospective case series of 18 patients with chronic proximal hamstring tendinopathy. Each patient received a single PRP injection by US guidance. Patients had chronic hamstring pain symp-

Table I. Clinical studies on PRP treatment for hip and pelvic pathologies.

\begin{tabular}{|c|c|c|c|c|}
\hline Study & $\begin{array}{l}\text { Level of } \\
\text { evidence }\end{array}$ & Pathology & Control group(s) & Outcomes measured \\
\hline A Hamid et al, $2014^{32}$ & II & $\begin{array}{l}\text { Acute hamstring } \\
\text { injuries }\end{array}$ & $\begin{array}{l}\text { Rehabilitation } \\
\text { without PRP }\end{array}$ & $\begin{array}{l}\text { Return to play time, pain severity } \\
\text { score, pain interference score }\end{array}$ \\
\hline Hamilton et al, $2015^{33}$ & I & Hamstring injuries & $\begin{array}{l}\text { Platelet-poor plasma, } \\
\text { no injection }\end{array}$ & Return to play time, reinjury rate \\
\hline Fader et al, $2015^{34}$ & IV & $\begin{array}{l}\text { Chronic proximal } \\
\text { hamstring tendinopathy }\end{array}$ & $\mathrm{N} / \mathrm{A}$ & VAS pain \\
\hline Wetzel et al, $2013^{35}$ & III & $\begin{array}{l}\text { Proximal hamstring } \\
\text { injuries }\end{array}$ & $\begin{array}{l}\text { NSAIDs, physical } \\
\text { therapy }\end{array}$ & VAS, NPRS \\
\hline Davenport et al, $2015^{36}$ & I & $\begin{array}{l}\text { Proximal hamstring } \\
\text { tendinopathy }\end{array}$ & $\begin{array}{l}\text { Autologous whole } \\
\text { blood }\end{array}$ & $\begin{array}{l}\text { MHHS, Hip Outcome Score-ADL, } \\
\text { IHOT-33 }\end{array}$ \\
\hline Dallaudière et al, $2014^{38}$ & IV & $\begin{array}{l}\text { Upper and lower limb } \\
\text { tendinopathy }\end{array}$ & $\mathrm{N} / \mathrm{A}$ & WOMAC, ultrasound lesion size \\
\hline Mautner et al, $2013^{41}$ & IV & Chronic tendinopathy & $\mathrm{N} / \mathrm{A}$ & $\begin{array}{l}\text { VAS, assessment of functional } \\
\text { pain, overall satisfaction }\end{array}$ \\
\hline Dallari et al, $2016^{16}$ & I & Hip OA & $H A, H A+P R P$ & VAS, HHS, WOMAC \\
\hline Battaglia et al, $2013^{44}$ & I & Hip OA & $\mathrm{HA}$ & HHS, VAS \\
\hline Redmond et al, $2015^{46}$ & II & Hip labral tears & Bupivacaine & $\begin{array}{l}\text { VAS, MHHS, Hip Outcome Score- } \\
\text { ADL, Hip Outcome Score-Sport- } \\
\text { Specific Subscale, Non-Arthritic } \\
\text { Hip Score, revision rate }\end{array}$ \\
\hline Martin et al, $2013^{55}$ & IV & AVN of femoral head & $\mathrm{N} / \mathrm{A}$ & Pain relief, failure rate \\
\hline
\end{tabular}

VAS=visual analog scale, NPRS=Nirschl Phase Rating Scale, MHHS=Modified Harris Hip Score, ADL=activities of daily living, IHOT-33=International Hip Outcome Tool 33, WOMAC=Western Ontario and McMaster Universities Osteoarthritis Index, $\mathrm{OA}=$ osteoarthritis, $\mathrm{HA}=$ hyaluronic acid, $\mathrm{HHS}=$ Harris Hip Score, AVN=avascular necrosis. 
toms for an average of 32.6 months prior to their injection and all patients had attempted other non-surgical treatments such as cortisone injections and physical therapy prior to injection. Based on a visual analog scale (VAS) for pain, patients had an average improvement in pain of $63 \%$ at 6 months following PRP injection.

In another study, Wetzel et al. ${ }^{35}$ performed a retrospective cohort study comparing twelve cases of proximal hamstring injuries treated by a single PRP injection and five patients treated with traditional conservative treatment (TCT) consisting of NSAIDs and physical therapy. Patients in the PRP and TCT groups presented at an average of 9.6 and 7.8 months after injury, respectively. At an average follow-up of 4.5 months, the PRP group demonstrated significantly improved VAS $(p<0.01)$ and Nirschl Phase Rating Scale (NPRS) scores $(p<0.01$ ) compared to pre-treatment. At an average follow-up of 2 months, the TCT group did not show the same degree of improvement in VAS $(p=0.06)$ or NPRS scores $(p=0.06)$. However, due to the small sample sizes and differences in follow-up times, it is difficult to discern these outcome differences.

Davenport et al. ${ }^{36}$ conducted a double-blind, randomized controlled trial comparing a single injection of PRP versus autologous whole blood (WB) for the treatment of proximal hamstring tendinopathy. At follow-up times of 2, 6, and 12 weeks and 6 months, no significant differences were observed between groups with regards to the Modified Harris Hip Score, Hip Outcome Scores for activities of daily living (ADL) and sport-specific function, and International Hip Outcome Tool 33 (IHOT33). However, compared to baseline, the PRP group demonstrated significant improvements in ADL and IHOT-33 scores, whereas the WB group showed significantly decreased pain with 15-minute sitting at 6 months.

Although conflicting results exist, the majority of published studies have reported successful outcomes of PRP injections for hamstring injuries. The Authors use PRP injections for acute hamstring injuries including, in rare cases, complete tears with a small amount of retraction in patients with low activity levels or when patients opt out of surgery.

\section{Adductor tendinopathy/athletic pubalgia}

Adductor tendinopathy typically presents with groin pain and is often seen in soccer players due to the frequency of running and cutting movements involved in this sport. ${ }^{37}$ Athletic pubalgia is a more general term involving groin pain, often in athletes, with adductor tendinopathy being a frequent concomitant pathology in these patients. Good outcomes have been shown following adductor tenotomy with and without hernioplasty ${ }^{37}$, though PRP provides a non-operative treatment option for this pathology.

Adductor longus tendinopathy is a common indication for PRP treatment. Dallaudière et al. ${ }^{38}$ performed a retrospective case series of 408 consecutive patients treated by a single ultrasound-guided PRP injection for tendinopathy of upper (medial and lateral epicondylar tendons, i.e. golfer's and tennis elbow, respectively) and lower (patellar, Achilles, hamstring, adductor longus, and peroneal tendons) limbs. Patients with hamstring and adductor longus tendinopathy demonstrated significantly improved Western Ontario and McMaster Universities Osteoarthritis Index (WOMAC) scores at 6 weeks and a mean 20.2 months following injection $(p<0.001)$. Ultrasound was also used to assess lesion size at baseline and 6 weeks post-injection, with hamstring and adductor longus tendon lesion size decreasing from an average of $21.2 \mathrm{~mm}$ to $2.6 \mathrm{~mm}$ during this time ( $p<0.001)$.

Interestingly, there is a high incidence of athletic pubalgia symptoms in patients with symptomatic femoroacetabular impingement $(\mathrm{FAI})^{39}$. Hammoud et al. ${ }^{39}$ showed that $39 \%$ of professional athletes presenting with concomitant $\mathrm{AP}$ and $\mathrm{FAl}$ experienced symptom resolution with surgical treatment of FAl alone. Larson et al. ${ }^{40}$ demonstrated a return to unrestricted activity in $89 \%$ of patients with surgical management of both athletic pubalgia and intra-articular hip pathologies, compared to only $25 \%$ in patients undergoing surgical treatment of athletic pubalgia alone.

The Authors inject the origin of the adductor longus (AL) tendon for chronic tendinopathy or acute tears. We first exclude the pubic symphysis as the source of the pain (using a lidocaine test) due to its anatomical proximity to the $A L$. It is also important to conduct full range of motion (ROM) evaluation of the hip joint to exclude concomitant FAI. FAI results in reduced ROM which in turn places increased stress on the $A L$ origin and may result in chronic microtears and tendinopathy. In these cases, PRP may not be a beneficial long-term solution as the offending mechanism is still present.

When electing to perform PRP injections for adductor tendinopathy, it is important to have the patient shave the groin area a few days prior to injection for ease of US guidance. These injections can be very painful, though due to the superficial location of the pathology, lidocaine cannot be used. It is recommended that nitric oxide be used as an inhaled anesthetic if possible.

\section{Gluteus medius tendinopathy}

The literature is currently lacking in reported outcomes of PRP injection for gluteus medius tendinopathy. However, in a multicenter, retrospective review of 180 patients with chronic tendinopathy ${ }^{41}, 16$ patients underwent US-guided PRP injections for gluteus medius tendinopathy with 13 patients demonstrating moderate improvement to complete resolution of symptoms at an average follow-up of 15 months post-injection. However, the PRP injection methodology of this study was non-uniform in that $60 \%$ of all patients received one injection, $30 \%$ received two, and $10 \%$ received three or more injections.

The Authors use a series of three injections of PRP for chronic indications ${ }^{8}$ such as gluteus medius or minimus tendinopathy. It is important to conduct a comprehensive physical examination to determine that the reported pain is not referred from the lower back or hip joint. In some cases, a lidocaine test can help to confirm or exclude the gluteus as the primary pathology. It is in- 
strumental to review a high resolution MRI of the region and to determine pre-injection if gluteal bursitis is causing the patient's pain or if tendinopathic changes/tears are present. It is also important to note which tendons are involved, as the minimus tendon is deeper and thus requires proper US guidance during injection. From our experience, the majority of gluteal tendon tears are in the medial/deeper side of the tendon.

As mentioned with hamstring tendinopathy, many practitioners would use steroid injections for greater trochanteric bursitis. However, this is usually a misdiagnosis or a secondary pathology as gluteal tendon tears are often the primary pain source. Although steroid injections can improve pain for a few months, they can also result in atrophy and weakening of the gluteal tendons.

\section{Hip osteoarthritis, acute inflammation, and labral tears}

As a conservative measure, osteoarthritis (OA) of the hip joint is commonly treated with intra-articular injections of corticosteroids or hyaluronic acid (HA). However, PRP has shown promise in reducing early-stage OA symptoms more effectively than $\mathrm{HA}^{6,42,43}$. Although less research is available on the efficacy of PRP for hip OA, the concept and mechanism of action are the same as in knee OA.

Two high quality studies have compared PRP versus HA for the treatment of hip OA. Recently, Dallari et al. ${ }^{16}$ performed a randomized, controlled trial on 111 patients to compare the efficacy of autologous PRP, HA, and a combination of both for the treatment of hip OA. Patients and health care providers were not blinded to the treatments used, though data collectors and analysts were blinded. Patients received three intra-articular US-guided injections one week apart during outpatient surgery, though the types of surgical procedures were not mentioned. In addition, the leukocyte concentration of the PRP formulations was not mentioned. Patients were assessed at 2,6 , and 12 months after treatment. The PRP group demonstrated lower VAS pain scores at all follow-up times and significantly better WOMAC scores at the 2- and 6-month follow-up periods.

Battaglia et al. ${ }^{44}$ also performed a non-blinded, randomized trial comparing US-guided PRP versus HA injections for hip OA in 100 consecutive patients. Patients underwent three injections every two weeks of 5 $\mathrm{mL}$ autologous PRP or $2 \mathrm{~mL}$ HA. The PRP samples were obtained through a double-spin technique to create a six-fold platelet count. Using the Harris Hip Score (HHS) and VAS, patients in both groups demonstrated significant improvements between 1- and 3-month follow-up. Although patients showed progressive worsening of symptoms between 6- and 12-month follow-up, scores were still significantly improved compared to baseline $(p<0.0005)$. No significant differences were found between the PRP and HA groups.

The Authors use bedside non-image-guided intra-articular hip injections through an anterior approach, as described previously ${ }^{45}$ using PRP. Following PRP injection, in order to promote circulation of the PRP through- out the joint, the patient is asked to lie for 30 seconds in the left lateral decubitus position, then 30 seconds prone, then 30 seconds in the right lateral decubitus position.

From our experience, PRP injections can help not only in cases of $\mathrm{OA}$ but also with high-level athletes who present with acute hip inflammation due to intra-articular pathology such as FAl-induced labral tear or ligamentum teres (LT) strain. In these cases, PRP improves inflammatory symptoms and enables a quick return to play without the possible negative effects of steroids. Athletes who have an underlying structural abnormality such as FAI typically elect to have a definitive solution in the form of arthroscopic surgery during the offseason, using PRP to enable continued play during the season. Redmond et al. ${ }^{46}$ recently performed a prospective cohort study to compare outcomes of intraoperative PRP versus bupivacaine injection for patients undergoing hip arthroscopy for labral tears. Patients receiving intraoperative PRP injections were found to have significantly higher (i.e. worse) pain scores and significantly lower modified Harris Hip Scores at 2 years postoperatively. However, no significant difference was found between groups in terms of conversion to total hip arthroplasty or revision surgery.

\section{Osteitis pubis.}

Osteitis pubis refers to inflammation of the pubic symphysis and surrounding muscle insertions. Typically, patients experience localized pain over the symphysis with radiation distally. From our experience, this is usually seen either in females following childbirth or in athletes with large Cam lesions and reduced ROM. Conservative management is typically initiated with a combination of rest, core stability exercises, and corticosteroid injections to the pubic symphysis. No published studies to date have sought to determine the efficacy of PRP for the treatment of osteitis pubis.

Prior to considering PRP injections into the pubic symphysis, the diagnosis of osteitis pubis must be confirmed. This can be done with a lidocaine injection under US to assess for temporary pain relief, though PRP should not be injected at the time of the lidocaine test. Other options include surgical treatment with debridement for males or pubic symphysis fusion for females.

\section{Muscle strains}

Muscle strains are a common injury, especially among athletes. Strains are typically graded from 1-3, with a grade 3 strain representing complete rupture of a muscle. Platelet-rich plasma has been used for the treatment of strains of the hamstrings, adductors, gluteus, iliopsoas, and external abdominal oblique, among other muscles ${ }^{47-49}$. The mechanism of the muscle strain may affect the efficacy of PRP injections. Using a rat model, Hammond et al. ${ }^{50}$ showed that repetitive, small strains may benefit more from PRP treatment than a single, large strain. Although the available evidence for the use of PRP to treat muscle strains is limited, PRP has been shown to reduce pain and swelling while reducing functional recovery time ${ }^{48}$.

A 19-year-old male college baseball player presented 
to our clinic with chronic left anterior thigh pain and reduced quadriceps strength. MRI demonstrated an $80 \%$ avulsion of the rectus femoris. Aspiration of $1 \mathrm{~mL}$ of seroma/hematoma was performed prior to two PRP injections in the left thigh (Figure 1). PRP injections were separated by nine days. The patient was placed in a locked hip and knee brace for several weeks to allow for healing after the injections. At nine weeks following the first injection, US demonstrated significant tissue formation in the area previously occupied by hematoma. On physical examination at this time, the patient's quadriceps volume was visibly larger with improved strength compared to pre-injection.

\section{Sartorius avulsion}

The sartorius originates on the anterior superior iliac spine (ASIS) apophysis, which begins to ossify between the ages of 13-15 years and fuses with the ilium between the ages of 21-25 years. An ASIS apophyseal avulsion injury occurs most commonly during running with the hip in extension and knee in flexion, or during a kicking motion ${ }^{51}$. Pain and a tearing sensation are the most common symptoms.

Recently, a 16 year old male presented to our clinic with anterolateral left hip pain following a lacrosse injury in which the patient made a cut while sprinting and felt and heard a pop around his hip. MRI showed a a- fproximal sartorius avulsion with a few millimeters of distraction and significant soft tissue and bone edema in the surrounding area. The patient underwent USguided PRP injection and reported complete resolution of symptoms within a week. He returned to full activity with our clearance at five weeks post-injection. A similar technique can be used for other apophyseal avulsion injuries such as that of the hamstrings or rectus femoris.

\section{Surgical applications}

As described above, when used in a non-operative setting, platelet-rich plasma is applied as a liquid injectable. However, in an intraoperative setting, PRP may be injected as a liquid or gel ${ }^{52}$, or delivered as a fibrin matrix (platelet-rich fibrin matrix, PRFM) ${ }^{53}$. Most of the surgical applications of PRP for hip and pelvic pathology are novel, with few studies currently in the literature.

\section{A vascular necrosis of the femoral head}

The use of PRP for augmentation of arthroscopic core decompression of avascular necrosis (AVN) of the femoral head has been described ${ }^{54}$. In patients with grade I or IIA AVN, the Authors perform core decom-

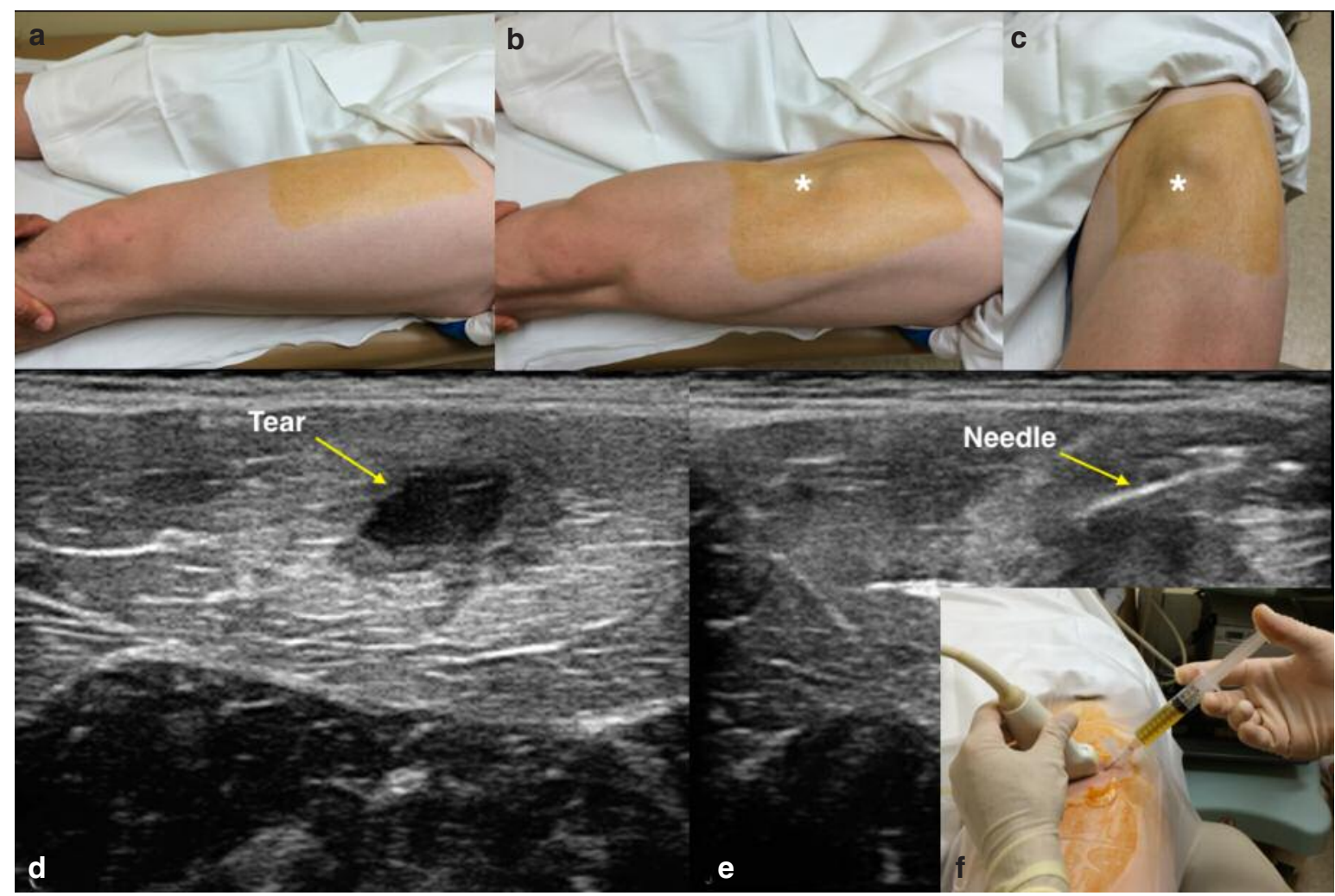

Figure 1 a-f. PRP for rectus femoris avulsion. a) Relaxed quadriceps muscles. b and c) Contracted quadriceps. *Denotes muscle belly defect due to tear. d) US image showing tear as hypoechoic region (yellow arrow). e) US image showing needle penetration into hypoechoic region to deliver PRP. f) Clinical photograph of US-guided PRP injection into rectus femoris tear. 
pression of necrotic areas of the femoral head via fluoroscopic guidance prior to inserting a trocar into the perforation hole and delivering $10 \mathrm{~mL}$ of liquid PRP (PRGF). In patients with grade IIB and IIC AVN, the Authors use an iliac bone graft which is mixed with activated PRP. The bone graft-PRP composite is then introduced into the drill track. Unfortunately, this surgical technique has not been investigated for patient outcomes.

In a separate study ${ }^{55}$, the use of a mixture of PRP and autologous bone marrow aspirate concentrate (BMAC) is injected into the site of necrosis following open decompression of the femoral head. In a case series of 73 hips with stage I $(n=57)$ or II $(n=16)$ AVN, the Authors reported that 16 hips progressed to further stages of AVN and ultimately required a total hip arthroplasty.

PRP has also been used as a non-surgical treatment option for AVN of the femoral head. In one case report, Pak et al. ${ }^{56}$ describe the injection of a mixture of PRP and autologous adipose-derived stem cells (ASCs) into the femoral head under US guidance. The procedure was repeated weekly for four weeks. The patient, a 43 year-old male with stage I AVN, showed significant improvement 3 months after treatment, with MRI demonstrating complete resolution of AVN.

Our group uses a similar technique to the one described by Guadilla ${ }^{54}$. In our small series of patients with grade I or IIA AVN of the femoral head, all patients had complete resolution of pain and resolution of the lesion on follow-up MRI (Figure 2).

\section{Hip microfracture}

Numerous studies have demonstrated the positive effects of PRP on cartilage repair and healing enhancement in various animal models ${ }^{57-60}$. More recently, PRP has also been used in clinical studies in patients with knee cartilage or meniscal degeneration, knee OA, and ankle cartilage defects ${ }^{8,61-63}$. However, no published studies have sought to determine the efficacy of PRP in augmenting microfracture surgery of the hip.

Based on the published evidence of PRP's positive effects on cartilage healing, we have recommended PRP injections to patients undergoing hip arthroscopy with cartilage damage, in particular to high-level athletes undergoing hip microfracture. Our functional and 6-to 12month follow-up MRI results indicate promising cartilage healing outcomes. In the short term, as is the case in knee-related studies, PRP reduces postoperative pain and expedites early return to function. Our technique includes 2-3 weekly injections starting 3-5 days postoperatively. We prefer to avoid intraoperative infiltration due to the dilution of the PRP concentrate by the saline used during arthroscopy.

\section{Pubic symphysis debridement}

Debridement of the pubic symphysis may be indicated in male athletes in whom significant degeneration of the symphysis is seen, usually following chronic stress from FAl-related reduced ROM. Surgery is indicated only after the pubic symphysis is confirmed as the source of pain (with a lidocaine test as described above) and

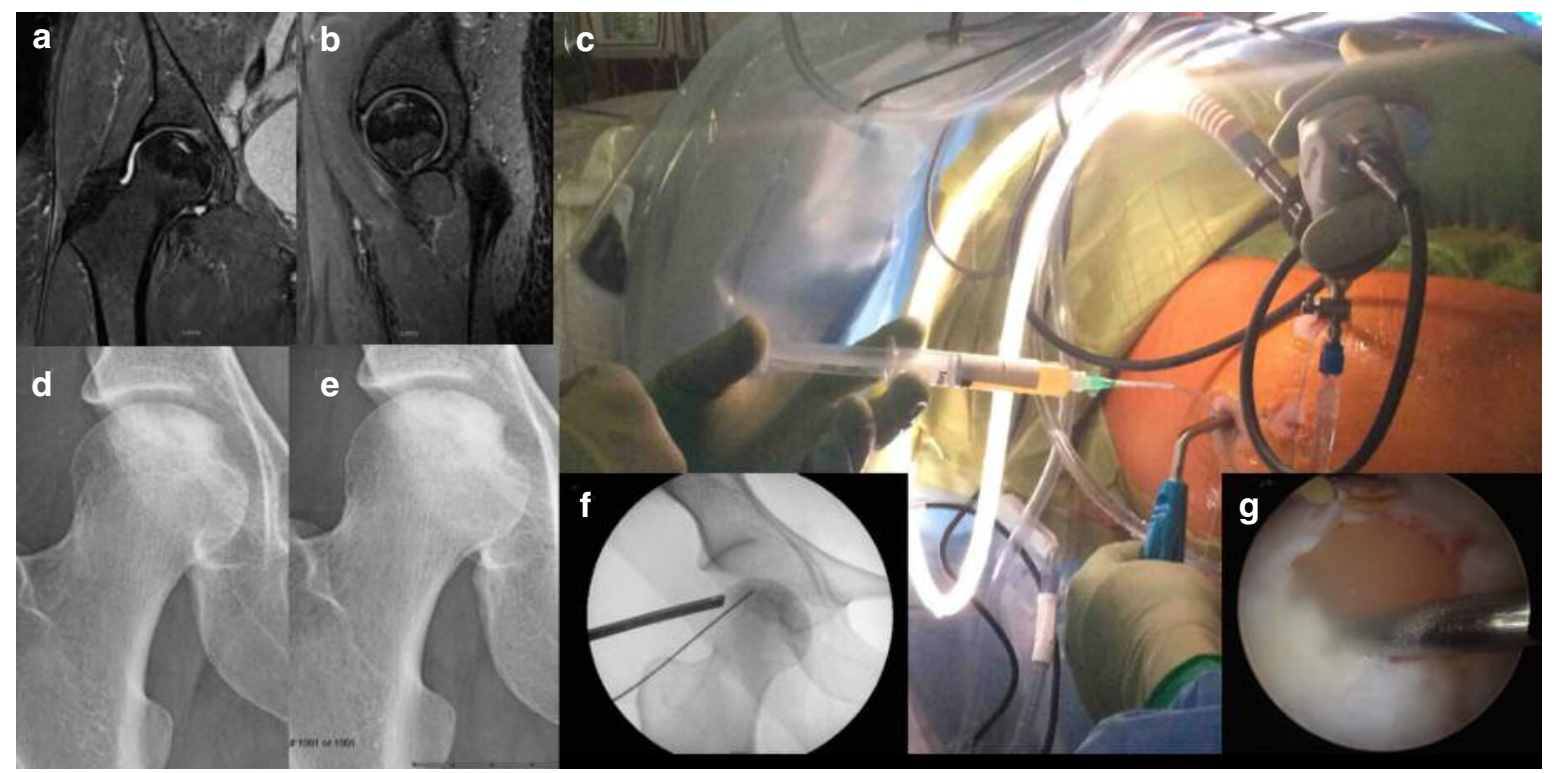

Figure 2 a-g. PRP for hip AVN. a) Coronal and b) sagittal proton density fat saturation MRI of the right hip showing Stage IIA AVN of the femoral head. c) Intraoperative photograph showing delivery of PRP through the mid-trochanteric portal using a slotted cannula. The arthroscope is inserted through the mid-anterior portal to visualize delivery of PRP. d) Preoperative radiograph of the right hip showing Stage IIA (pre-collapse) AVN of the femoral head. e) Postoperative radiograph showing improved density within the cystic AVN lesion in the femoral head following PRP injection. f) Intraoperative fluoroscopic view showing targeting of needle within AVN lesion for PRP delivery. g) Arthroscopic view of PRP delivery into the femoral head. 
imaging indicates early osteoarthritic changes. MRI may demonstrate significant bone edema on both sides of the joint, while computed tomography (CT) may show subchondral cysts and bone sclerosis. No previous studies have sought to determine the efficacy of PRP for augmenting pubic symphysis debridement. A minimally invasive approach is used to enter the joint via the proximal and superoanterior capsule. Debridement of a few millimeters of degenerated cartilage and subchondral bone from both sides of the joint is performed. Care is taken to scrape all cartilaginous tissue including the inferior portion of the joint which tends to be difficult due to limited space. Fluoroscopy can confirm the location of bone rasps used for this part of the procedure and to determine the gap achieved between the two sides of the joint. Before closing, Gelfoam (Pfizer, New York City, NY) is soaked in PRP concentrate until it becomes more solid in form, at which time it is placed at the gap between the bones (Figure 3). This reduces postoperative symptoms and generates rapid healing of bridging tissue, including enhancement of the joint's capsular tissue regeneration. After complete bed rest for a few days, patients are to avoid aggravating activities due to the surgical approach requiring separation of the lower abdominal muscles. Patients may begin return to activity 2.5 to 3 months following surgery, with eventual return to full level activity.

\section{Adjuvant therapy for tendon repair}

Platelet-rich plasma has been shown to improve healing in patients with acute ruptured Achilles tendons ${ }^{11,64}$ Sánchez et al. ${ }^{64}$ showed an earlier recovery of range of motion, return to gentle running, and return to training activities in 12 athletes who underwent open suture repair of a complete Achilles tendon rupture with PRP versus standard open suture repair. Alsousou ${ }^{11} \mathrm{com}-$ pared biopsy samples of acute ruptured Achilles tendons treated with PRP versus a control group receiving no treatment. The PRP group demonstrated significantly higher collagen I content and a significantly lower modified Bonar score, which indicates improved early tendon healing.

The Authors use PRP injections in patients undergoing surgical repair of the hamstring tendons when the ten-
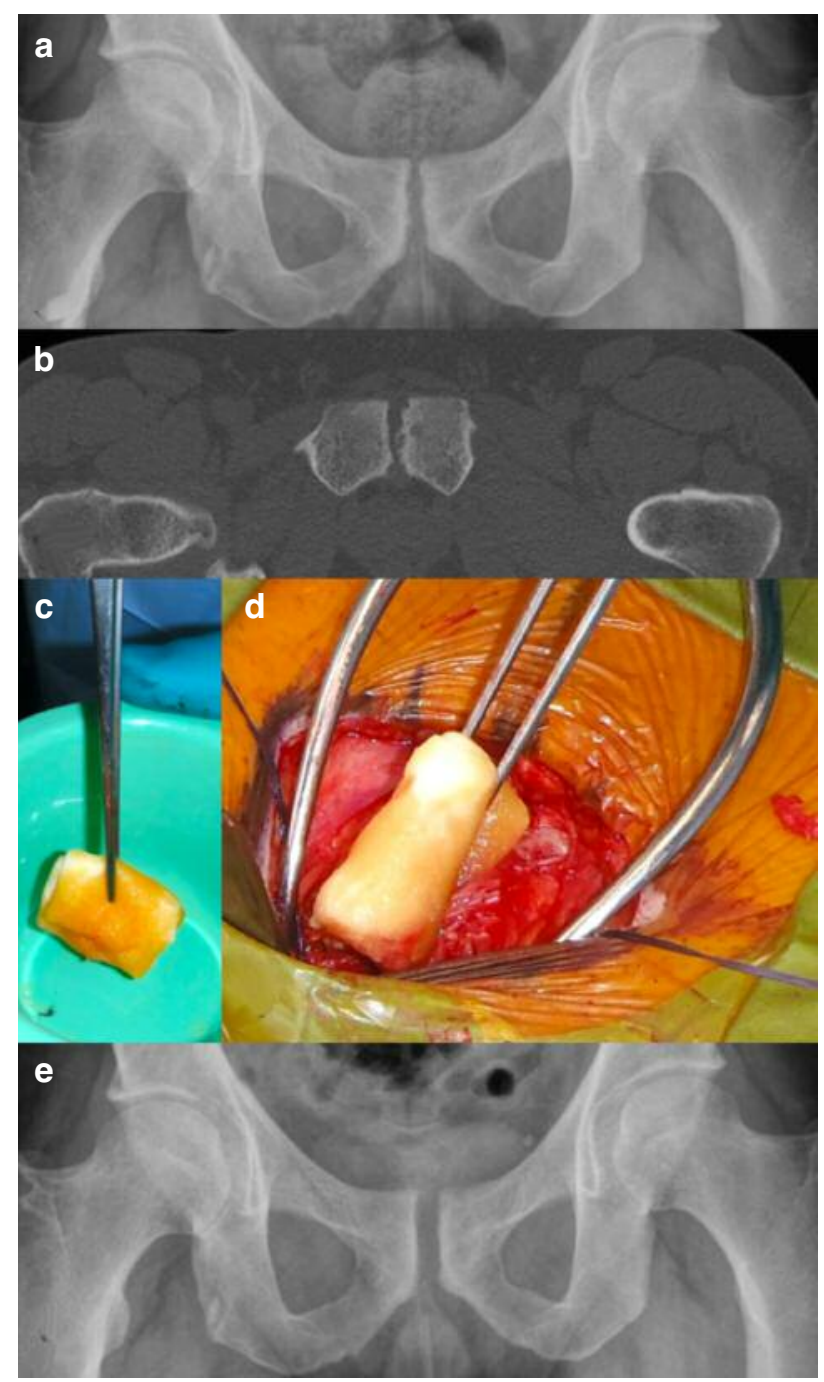

Figure 3 a-e. PRP for pubic symphysis debridement. a) Anteroposterior (AP) radiograph of the pelvis showing osteitis pubis. Irregularity in the pubic symphysis with osteophytes, joint space narrowing, and sclerosis. b) Axial CT scan of pelvis showing subchondral cysts and osteophytes along with joint space narrowing in the pubic symphysis. c) Gelfoam soaked in PRP. d) Gelfoam soaked in PRP being inserted into pubic symphysis following debridement. e) Postoperative radiograph following debridement of pubic symphysis. Note the wider joint space with smooth margins. 
don tissue is found to be of low quality and enhancement of the surgical repair is required. Another common indication for PRP within our practice is in professional athletes for whom there is a need for an expedited recovery and return to play. This is most commonly indicated for the hamstrings, rectus femoris, or a sartorius avulsion.

\section{Conclusions}

This review describes some of the established as well as novel applications of platelet-rich plasma for the treatment of hip and pelvic pathologies. Although the outcomes of many of these applications have not been described in the literature, particularly in high-level studies, from our experience we have found that symptomatic and functional outcomes are successful in the majority of patients. As indications for PRP continue to expand, it will become increasingly important for future studies to state specific methodologies used in the preparation of PRP in order to recognize ideal preparation techniques and the ideal number of PRP injections for each pathology. Leukocyte-poor PRP has the advantages of a reduced inflammatory response and mainly anabolic changes compared to leukocyte-rich $\mathrm{PRP}$, though further, high quality studies are necessary to determine outcome differences between these two PRP preparations.

\section{References}

1. Mei-Dan O, Carmont MR. Novel applications of platelet-rich plasma technology in musculoskeletal medicine and surgery. Oper Tech Orthop. 2012;22:56-63.

2. DeLong JM, Russell RP, Mazzocca AD. Platelet-rich plasma: the PAW classification system. Arthroscopy. 2012;28:9981009.

3. Castricini R, Longo UG, De Benedetto M, et al. Platelet-rich plasma augmentation for arthroscopic rotator cuff repair: a randomized controlled trial. Am J Sports Med. 2011;39:258-265.

4. Mei-Dan O, Carmont MR. Autologous blood products in rotator cuff repair. Med Sport Sci. 2012;57:65-75.

5. Liddle AD, Rodríguez-Merchán EC. Platelet-rich plasma in the treatment of patellar tendinopathy: A systematic review. Am J Sports Med. 2015;43:2583-2590.

6. Meheux CJ, McCulloch PC, Lintner DM, Varner KE, Harris JD. Efficacy of intra-articular platelet-rich plasma injections in knee osteoarthritis: A systematic review. Arthroscopy. 2016;32:495505.

7. Mishra AK, Skrepnik NV, Edwards SG, et al. Efficacy of platelet-rich plasma for chronic tennis elbow: a double-blind, prospective, multicenter, randomized controlled trial of $230 \mathrm{pa}-$ tients. Am J Sports Med. 2014;42:463-471.

8. Mei-Dan O, Carmont MR, Laver L, Mann G, Maffulli N, Nyska M. Platelet-rich plasma or hyaluronate in the management of osteochondral lesions of the talus. Am J Sports Med. 2012 40:534-541.

9. Kajikawa $\mathrm{Y}$, Morihara $\mathrm{T}$, Sakamoto $\mathrm{H}$, et al. Platelet-rich plasma enhances the initial mobilization of circulation-derived cells for tendon healing. J Cell Physiol. 2008;215:837-845.

10. Visser LC, Arnoczky SP, Caballero O, Kern A, Ratcliffe A, Gardner KL. Growth factor-rich plasma increases tendon cell proliferation and matrix synthesis on a synthetic scaffold: an in vitro study. Tissue Eng Part A. 2010;16:1021-1029.

11. Alsousou J, Thompson M, Harrison P, Willett K, Franklin S. Effect of platelet-rich plasma on healing tissues in acute ruptured Achilles tendon: a human immunohistochemistry study. Lancet. 2015;385 Suppl 1:S19.

12. Virchenko $O$, Aspenberg P. How can one platelet injection after tendon injury lead to a stronger tendon after 4 weeks? Interplay between early regeneration and mechanical stimulation. Acta Orthop. 2006;77:806-812.

13. Mei-Dan O, Lippi G, Sánchez M, Andia I, Maffulli N. Autologous platelet-rich plasma: a revolution in soft tissue sports injury management? Phys Sportsmed. 2010;38:127-135.

14. Lee JW, Kwon OH, Kim TK, et al. Platelet-rich plasma: quantitative assessment of growth factor levels and comparative analysis of activated and inactivated groups. Arch Plast Surg. 2013:40:530-535.

15. Kakudo N, Morimoto N, Kushida S, Ogawa T, Kusumoto K. Platelet-rich plasma releasate promotes angiogenesis in vitro and in vivo. Med Mol Morphol. 2014;47:83-89.

16. Dallari D, Stagni C, Rani N, et al. Ultrasound-guided injection of platelet-rich plasma and hyaluronic acid, separately and in combination, for hip osteoarthritis: A randomized controlled study. Am J Sports Med. 2016;44:664-671.

17. Padulo J, Oliva F, Frizziero A, Maffulli N. Muscles, Ligaments and Tendons Journal. Basic principles and recommendations in clinical and field science research: 2016 Update. MLTJ. 2016;6(1):1-5.

18. Samuelson EM, Odum SM, Fleischli JE. The cost-effectiveness of using platelet-rich plasma during rotator cuff repair: A Markov model analysis. Arthroscopy. 2016;32:1237-1244.

19. Dhurat R, Sukesh M. Principles and methods of preparation of platelet-rich plasma: A review and author's perspective. J Cutan Aesthet Surg. 2014;7:189-197.

20. Arnoczky SP, Sheibani-Rad S. The basic science of plateletrich plasma (PRP): what clinicians need to know. Sports Med Arthrosc. 2013;21:180-185.

21. Ghassab S, Dulin J, Bertone AL. Thromboelastographic clot characteristics of autologous equine blood products after activation by autologous thrombin, bovine thrombin, or calcium chloride. Vet Surg. 2015;44:970-975.

22. Textor JA, Tablin F. Activation of equine platelet-rich plasma: comparison of methods and characterization of equine autologous thrombin. Vet Surg. 2012;41:784-794.

23. Graziani F, Ivanovski S, Cei S, Ducci F, Tonetti M, Gabriele M. The in vitro effect of different PRP concentrations on osteoblasts and fibroblasts. Clin Oral Implants Res. 2006;17: 212-219.

24. Weibrich G, Hansen T, Kleis W, Buch R, Hitzler WE.. Effect of platelet concentration in platelet-rich plasma on peri-implant bone reintegration. Bone. 2004;34:665-671.

25. Anitua E, Sánchez M, Nurden AT, Nurden P, Orive G, Andía I. New insights into and novel applications for platelet-rich fibrin therapies. Trends Biotechnol. 2006;24:227-234.

26. Weibrich G, Kleis WK, Hitzler WE, Hafner G. Comparison of the platelet concentrate collection system with the plasmarich-in-growth-factors kit to produce platelet-rich plasma: a technical report. Int J Oral Maxillofac Implants. 2005;20:118123.

27. Cavallo C, Filardo G, Mariani E, et al. Comparison of plateletrich plasma formulations for cartilage healing: an in vitro study. J Bone Joint Surg Am. 2014;96:423-429.

28. Dragoo JL, Braun HJ, Durham JL, et al. Comparison of the acute inflammatory response of two commercial platelet-rich plasma systems in healthy rabbit tendons. Am J Sports Med. 2012;40:1274-1281.

29. McCarrel TM, Minas T, Fortier LA. Optimization of leukocyte concentration in platelet-rich plasma for the treatment of tendinopathy. J Bone Joint Surg Am. 2012;94:e143(1-8). 
30. Zhou Y, Zhang J, Wu H, Hogan MV, Wang JH. The differential effects of leukocyte-containing and pure platelet-rich plasma (PRP) on tendon stem/progenitor cells - implications of PRP application for the clinical treatment of tendon injuries. Stem Cell Res Ther. 2015;6:173.

31. Schippinger G, Prüller F, Divjak M, et al. Autologous plateletrich plasma preparations: Influence of nonsteroidal anti-inflammatory drugs on platelet function. Orthop J Sports Med 2015;3:2325967115588896.

32. A Hamid MS, Mohamed Ali MR, Yusof A, George J, Lee LP. Platelet-rich plasma injections for the treatment of hamstring injuries: a randomized controlled trial. Am J Sports Med. 2014;42:2410-2418.

33. Hamilton B, Tol JL, Almusa E, et al. Platelet-rich plasma does not enhance return to play in hamstring injuries: a randomised controlled trial. Br J Sports Med. 2015;49:943-950.

34. Fader R, Mitchell JJ, Traub S, et al. Platelet-rich plasma treatment improves outcomes for chronic proximal hamstring injuries in an athletic population. Muscles Ligaments Tendons $\mathrm{J}$. 2015;4:461-466.

35. Wetzel RJ, Patel RM, Terry MA. Platelet-rich plasma as an effective treatment for proximal hamstring injuries. Orthopedics. 2013;36:e64-e70.

36. Davenport KL, Campos JS, Nguyen J, Saboeiro G, Adler RS, Moley PJ. Ultrasound-guided intratendinous injections with platelet-rich plasma or autologous whole blood for treatment of proximal hamstring tendinopathy: A double-blind randomized controlled trial. J Ultrasound Med. 2015;34:14551463.

37. Mei-Dan O, Lopez V, Carmont MR, et al. Adductor tenotomy as a treatment for groin pain in professional soccer players. Orthopedics. 2013;36:e1189-e1197.

38. Dallaudière $B$, Pesquer L, Meyer $P$, et al. Intratendinous injection of platelet-rich plasma under US guidance to treat tendinopathy: a long-term pilot study. J Vasc Interv Radiol. 2014;25:717-723.

39. Hammoud S, Bedi A, Magennis E, Meyers WC, Kelly BT. High incidence of athletic pubalgia symptoms in professional athletes with symptomatic femoroacetabular impingement. Arthroscopy. 2012;28:1388-1395.

40. Larson CM, Pierce BR, Giveans MR. Treatment of athletes with symptomatic intra-articular hip pathology and athletic pubalgia/sports hernia: a case series. Arthroscopy. 2011;27:768775.

41. Mautner K, Colberg RE, Malanga G, et al. Outcomes after ultrasound-guided platelet-rich plasma injections for chronic tendinopathy: a multicenter, retrospective review. PM R. 2013:5:169-175.

42. Görmeli G, Görmeli CA, Ataoglu B, Çolak C, Aslantürk O, Ertem K. Multiple PRP injections are more effective than single injections and hyaluronic acid in knees with early osteoarthritis: a randomized, double-blind, placebo-controlled trial. Knee Surg Sports Traumatol Arthrosc. 2015. [Epub ahead of print].

43. Kilincoglu V, Yeter A, Servet E, Kangal M, Yildirim M. Short term results comparison of intraarticular platelet-rich plasma (prp) and hyaluronic acid (ha) applications in early stage of knee osteoarthritis. Int J Clin Exp Med. 2015;8:18807-18812.

44. Battaglia M, Guaraldi F, Vannini F, et al. Efficacy of ultrasoundguided intra-articular injections of platelet-rich plasma versus hyaluronic acid for hip osteoarthritis. Orthopedics. 2013;36: e1501-e1508.

45. Mei-Dan O, McConkey MO, Petersen B, McCarty E, Moreira B, Young DA. The anterior approach for a non-image-guided intra-articular hip injection. Arthroscopy. 2013;29:1025-1033.

46. Redmond JM, Gupta A, Stake CE, Hammarstedt JE, Finch NA, Domb BG. Clinical results of hip arthroscopy for labral tears: a comparison between intraoperative platelet-rich plas- ma and bupivacaine injection. Arthroscopy. 2015;31:445-453.

47. Dauty M, Menu P, Dubois C. Uncommon external abdominal oblique muscle strain in a professional soccer player: a case report. BMC Res Notes. 2014;7:684.

48. Hamilton BH, Best TM. Platelet-enriched plasma and muscle strain injuries: challenges imposed by the burden of proof. Clin J Sports Med. 2011;21:31-36.

49. Hamilton B, Knez W, Eirale C, Chalabi H. Platelet enriched plasma for acute muscle injury. Acta Orthop Belg. 2010;76: 443-448.

50. Hammond JW, Hinton RY, Curl LA, Muriel JM, Lovering RM. Use of autologous platelet-rich plasma to treat muscle strain injuries. Am J Sports Med. 2009;37:1135-1142.

51. Boyd KT, Peirce NS, Batt ME. Common hip injuries in sport. Sports Med. 1997;24:273-288.

52. Cervellin M, de Girolamo L, Bait C, Denti M, Volpi P. Autologous platelet-rich plasma gel to reduce donor-site morbidity after patellar tendon graft harvesting for anterior cruciate ligament reconstruction: a randomized, controlled clinical study. Knee Surg Sports Traumatol Arthrosc. 2012;20:114-120.

53. Rodeo SA, Delos D, Williams RJ, Adler RS, Pearle A, Warren RF. The effect of platelet-rich fibrin matrix on rotator cuff tendon healing: a prospective, randomized clinical study. Am J Sports Med. 2012;40:1234-1241.

54. Guadilla J, Fiz N, Andia I, Sánchez M. Arthroscopic management and platelet-rich plasma therapy for avascular necrosis of the hip. Knee Surg Sports Traumatol Arthrosc. 2012;20: 393-398.

55. Martin JR, Houdek MT, Sierra RJ. Use of concentrated bone marrow aspirate and platelet rich plasma during minimally invasive decompression of the femoral head in the treatment of osteonecrosis. Croat Med J. 2013;54:219-224.

56. Pak J, Lee JH, Jeon JH, Lee SH. Complete resolution of avascular necrosis of the human femoral head treated with adipose tissue-derived stem cells and platelet-rich plasma. J Int Med Res. 2014;42:1353-1362.

57. Kazemi D, Fakhrjou A. Leukocyte and platelet rich plasma (LPRP) versus leukocyte and platelet rich fibrin (L-PRF) for articular cartilage repair of the knee: A comparative evaluation in an animal model. Iran Red Crescent Med J. 2015;17:e19594.

58. Smyth NA, Haleem AM, Ross KA, et al. Platelet-rich plasma may improve osteochondral donor site healing in a rabbit model. Cartilage. 2016;7:104-111.

59. Sun $Y$, Feng $Y$, Zhang $C Q$, Chen SB, Cheng $X G$. The regenerative effect of platelet-rich plasma on healing in large osteochondral defects. Int Orthop. 2010;34:589-597.

60. Zhou Q, Xu C, Cheng X, et al. Platelets promote cartilage repair and chondrocyte proliferation via ADP in a rodent model of osteoarthritis. Platelets. 2016:27:212-222.

61. Duif C, Vogel T, Topcuoglu F, Spyrou G, von Schulze Pellengahr C, Lahner M. Does intraoperative application of leukocyte-poor platelet-rich plasma during arthroscopy for knee degeneration affect postoperative pain, function and quality of life? A 12-month randomized controlled double-blind trial. Arch Orthop Trauma Surg. 2015;135:971-977.

62. Filardo G, Kon E, Pereira Ruiz MT, et al. Platelet-rich plasma intra-articular injections for cartilage degeneration and osteoarthritis: single- versus double-spinning approach. Knee Surg Sports Traumatol Arthrosc. 2012;20:2082-2091.

63. Vannini F, Di Matteo B, Filardo G. Platelet-rich plasma to treat ankle cartilage pathology - from translational potential to clinical evidence: a systematic review. J Exp Orthop 2015;2:2.

64. Sánchez M, Anitua E, Azofra J, Andía I, Padilla S, Mujika I. Comparison of surgically repaired Achilles tendon tears using platelet-rich fibrin matrices. Am J Sports Med. 2007;35:245251. 